\title{
Persistent ascites due to sclerosing encapsulating peritonitis mimicking ovarian carcinoma: A case report
}

\author{
Mete Çağlar ${ }^{1}$, Nilüfer Çetinkaya², Emre Özgü², Tayfun Güngör ${ }^{2}$ \\ 'Department of Obstetrics and Gynecology, Düzce University Faculty of Medicine, Düzce, Turkey \\ 2Department of Gynecologic Oncology, Zekai Tahir Burak Women's Health Education and Research Hospital, Ankara, Turkey
}

\begin{abstract}
Sclerosing encapsulating peritonitis, also known as 'Cocoon Syndrome', is a rare cause of bowel obstruction. The condition might be congenital or acquired and has non-specific symptomatology. Abdominal pain occurs due to the limitation of intestinal motility or segment obstruction by a thick homogenous fibrotic mantle covering the intra-peritoneal organs. Altered peritoneal fluid dynamics result in persistent ascites. Leading pathogenic theories are not well defined, but genetic factors, retrograde trans-tubal flow of causative agents, peritoneal infections, medications and peritoneal invasive procedures are all thought to play a role. There are no specific diagnostic criteria and exact diagnosis is only confirmed during surgery when the investing thick fibrous folds covering the bowel loops are visualised. We present here a case that had been suspected to have an ovarian malignancy due to a huge abdominal heterogeneous mass and ascites on preoperative diagnostic workup, but had a final diagnosis of abdominal Cocoon Syndrome made during surgery. (J Turk Ger Gynecol Assoc 2014; 15: 201-3)
\end{abstract}

Key words: Abdominal Cocoon Syndrome, sclerosing encapsulating peritonitis, ascites

Received: 08 August, 2013

Accepted: 02 November, 2013

Available Online Date: 08 August, 2014

\section{Introduction}

'Abdominal Cocoon Syndrome' is characterised by the presence of thick homogenous fibrotic mantle covering the intraperitoneal organs. The condition can be either congenital or acquired. There is no specific symptomatology related with the disease. The exact cause has not been well studied, but genetic factors, trans-tubal flow of causative agents, peritoneal infections, medications and peritoneal invasive procedures are all thought to play a role (1).

Encapsulating membrane in abdominal Cocoon Syndrome is composed of dense fibrotic tissue and has no mesothelial cell covering (2). Cocoon Syndrome causes the disordered peritoneal fluid dynamics due to restricted bowel movements and the presence of a thick fibrous mantle surrounding the intestinal loops. These factors lower the trans-mural lymph flow and result in persistent ascites.

\section{Case Presentation}

A thirty-six year old, gravida 1, parity 1 female was admitted to a gynaecology clinic one year previously due to secondary infertility. She had no chronic illnesses in her past medical history. She gave birth six years ago by caesarean section after spontaneous pregnancy. Upon admission, she had menstrual regularity and hormonal evaluation was within normal limits. On trans-vaginal ultrasonography she had a normal appearing uterus and adnexa with ascites in the pouch of Douglas.
Trans-abdominal ultrasonography revealed grade I hepatosteatosis and abdominal ascites that was measured as $8 \mathrm{~cm}$ at the deepest point with free fluid accumulation surrounding the bladder and in between the bowel loops. Tumour markers including carcinoembryonic antigen (CEA), CA 125, CA 19-9, CA 15-3 and alpha fetoprotein (AFP) were normal and the patient was sent to the gastroenterology department for a differential diagnosis for ascites. She had normal liver function tests and albumin levels upon admission. Colour Doppler evaluations of the portal venous system and inferior vena cava were performed. On Doppler sonography, portal venous branches, splenic vein, inferior vena cava and hepatic veins had normal calibres and flow patterns. Also, the presence of luminal thrombi was excluded. Liver architecture was normal with no suspicious lesions and ascites was measured as 12 $\mathrm{cm}$ at its deepest point. Paracentesis revealed the presence of lymphocytic, histiocytic cells and mesothelial proliferation without atypia. Gastrointestinal malignancy was excluded due to these investigations. The patient was advised to return for re-evaluation after 3 months. However, the patient did not return for the follow-up.

The same patient was then admitted to our clinic one year later with the desire to become pregnant. She described pelvic pain during menses for 3 months. Her Pap test and hormonal profile were normal. During transvaginal ultrasonography, a 20x18 cm abdominal heterogeneous solid-cystic mass undifferentiated from the left adnexa with ascites was diagnosed. Tumour markers were normal. The patient was 


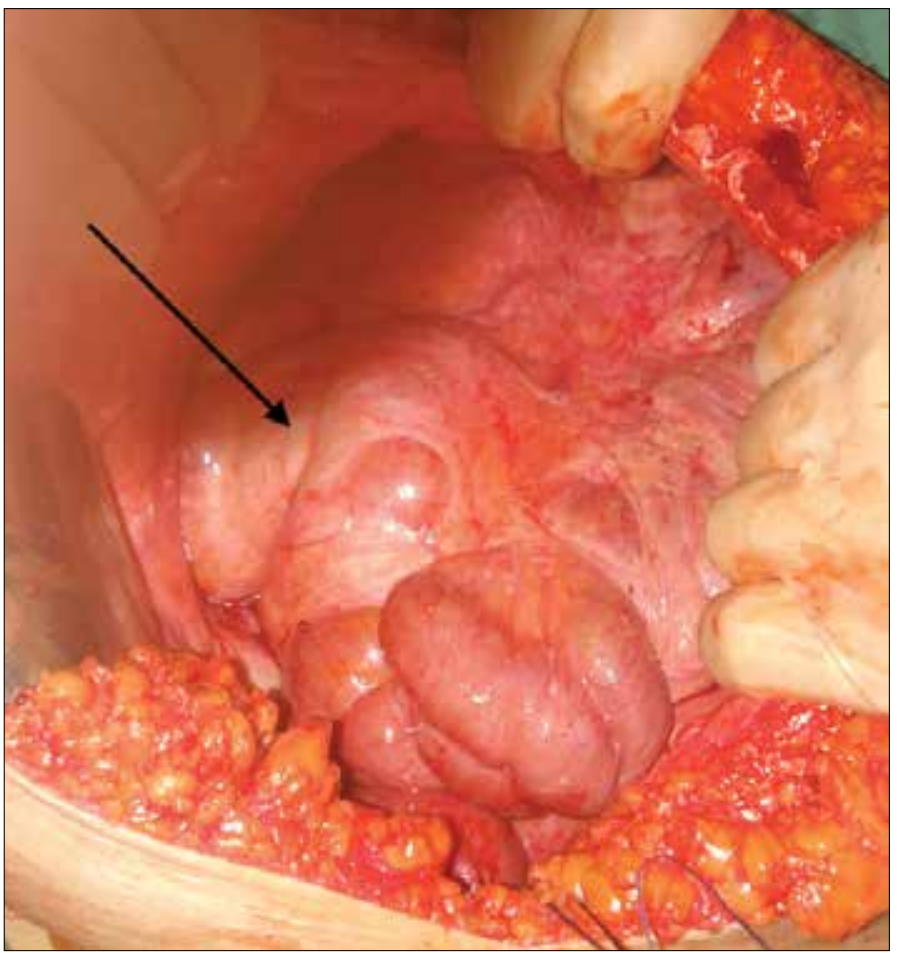

Figure 1. Arrow shows the thick fibrous mantle surrounding the bowel loops. Liver, stomach and spleen are also located within this fibrous capsule

prepared for diagnostic laparotomy with the probability of ovarian carcinoma due to new onset pelvic pain, a huge abdominal mass and ascites.

During the operation, about $2500 \mathrm{~mL}$ clear ascites was removed after peritoneal entry and thick fibrotic tissue encapsulating the abdominal organs including the liver, stomach, spleen, but excluding the uterus and adnexa, was observed (Figure 1). There was also an $8 \mathrm{~cm}$, benign cyst on the left ovary. The diagnosis of sclerosing encapsulating peritonitis was made during surgery and the operation was terminated following left ovarian cystectomy with the absence of malignancy on frozen section. The patient was discharged from the hospital on the third postoperative day without any complications. The permanent pathology result was reported one week later as a benign ovarian serous cyst. The patient was scheduled for evaluation in the infertility clinic 3 months after surgery and written informed consent was obtained from the patient to present her medical history in this study.

\section{Discussion}

Peritoneal fluid dynamic depends on diaphragmatic lymph flow and desired effects of bowel movements. Peritoneal fluid drains primarily to the upper abdominal, diaphragmatic lymph nodes and then continues via the thoracic duct to the mediastinum (3).

Diaphragmatic movements during inspiration and expiration, bowel peristalsis and impacts of abdominal wall musculature affect the peritoneal fluid dynamics. Blood and lymphatic ves- sels along the bowel wall also affect the absorption of peritoneal fluid with their hydrostatic and oncotic pressures.

Portal hypertension due to cirrhosis, hepatic congestion or portal thrombus formation is the primary cause of ascites. Hypoalbuminaemia, which leads to decreased plasma oncotic pressure, is also an important issue in the aetiology of ascites. Furthermore, peritoneal disease, medical disorders and malignant conditions are known to be associated with ascites. Intractable ascites is a term that reflects the presence of ascites in spite of strict sodium restriction and the maximal diuretic dose of furosemide and spironolactone (4).

Abdominal Cocoon Syndrome frequently presents with vague abdominal symptoms that do not clearly indicate a known aetiology. Bowel obstruction, abdominal mass, ascites and abdominal pain are the most common clinical presentations related with this syndrome.

Most of the patients are known to have symptoms associated with peritoneal tuberculosis, peritoneal dialysis, sarcoidosis, systemic lupus erythematosus, $\beta$-blocker use, cirrhosis and ventriculoperitoneal shunts (5). Patients are usually diagnosed per-operatively but procedures like ultrasonography or computed tomography (CT) have preoperative diagnostic roles. CT is known to be the gold standard diagnostic work-up due to the ease and feasibility of luminal assessment (1). Oral contrast studies might also present bowel obstructions which facilitate therapeutic interventions (6). In patients with bowel obstruction, the goal of treatment consists of resection of fibrous coverage and lysis of adhesions between the bowel loops to free the entire gut (7). Bowel resection is rarely indicated and necessary in the presence of highly oedematous, obstructive or necrotic segments.

In our patient, we did not see any symptoms pointing to the bowel obstruction preoperatively. The diagnosis of abdominal mass and persistent ascites for a period longer than a year in the presence of new onset pelvic pain was the main indication for surgery. The diagnosis of Cocoon Syndrome was made at the time of surgery when the ascites was drained. Lysis of the fibrous mantle was not performed in our case due to the absence of any bowel obstruction.

This case report represents a rare abdominal Cocoon Syndrome with persistent ascites and an accompanying left ovarian serous cyst. Our aim is to ensure that clinicians keep this rare condition in mind when faced with the presence of persistent ascites without any concomitant organ pathologies. Also, preoperative findings might indicate this syndrome, perhaps via a mixture of large cystic and solid masses with different degrees of swinging features in its core during ultrasonography. To date, the differential diagnosis of this condition consists of the exclusion of other aetiologies with ascites and measurements of tumour markers to reject probable malignant diseases. Females with abdominopelvic pain, ascites and an adnexal mass that is suspicious of ovarian malignancy, especially in the presence of negative tumour markers, might require attention in gynaecology clinics for the differential diagnosis of abdominal Cocoon Syndrome.

Ethics Committee Approval: Ethics committee approval was received for this study.

Informed Consent: Written informed consent was obtained from patient who participated in this study. 
Peer-review: Externally peer-reviewed.

Author contributions: Concept - M.C., N.C., E.O., T.G.; Design - M.C., N.C., E.O., T.G.; Supervision M.C., N.C., E.O., T.G.; Resource - M.C., N.C., E.O., T.G.; Materials - M.C., N.C., E.O., T.G.; Data Collection\&/or Processing - M.C., N.C., E.O., T.G.; Analysis\&/or Interpretation - M.C., N.C., E.O., T.G.; Literature Search - M.C., N.C., E.O., T.G.; Writing - M.C., N.C., E.O., T.G.; Critical Reviews - M.C., N.C., E.O., T.G.

Conflict of Interest: No conflict of interest was declared by the authors. Financial Disclosure: The authors declared that this study has received no financial support.

\section{References}

1. Solak A, Solak İ. Abdominal cocoon syndrome: Preoperative diagnostic criteria, good clinical outcome with medical treatment and review of the literature. Turk J Gastroenterol 2012; 23: 776-9.
2. Yeniay L, Karaca CA, Calışkan C, Fırat O, Ersin SM, Akgün E. Abdominal cocoon syndrome as a rare cause of mechanical bowel obstruction: Report of two cases. Ulus Travma Acil Cerrahi Derg 2011; 17: 557-60. [CrossRef]

3. Parungo CP, Soybel DI, Colson YL, Kim SW, Ohnishi S, DeGrand AM, et al. Lymphatic drainage of the peritoneal space: a pattern dependent on bowel lymphatics. Ann Surg Oncol 2007; 14: 286-98. [CrossRef]

4. Zervos EE, Rosemurgy AS. Management of medically refractory ascites. Am J Surg 2001; 181: 256-64. [CrossRef]

5. Singh B, Gupta S. Abdominal cocoon: A case series. Int J Surg 2013;11: 325-8. [CrossRef]

6. Wei B, Wei HB, Guo WP, Zheng ZH, Huang Y, Hu BG, Huang JL. Diagnosis and treatment of abdominal cocoon: a report of 24 cases. Am J Surg 2009; 198: 348-53. [CrossRef]

7. Chew MH, Sophian Hadi I, Chan G, Ong HS, Wong WK. A problem encapsulated: the rare peritoneal encapsulation syndrome. Singapore Med J 2006; 47: 808-10. 